\title{
Proporção de anemia de acordo com o estadiamento puberal, segundo dois critérios diagnósticos
}

\section{Anemia proportion according to pubertal stage using two diagnostic criteria}

\author{
Flávia Calanca da SILVA ${ }^{1}$ \\ Maria Sylvia de Souza VITALLE' \\ Elaine Cristina QUAGLIA' \\ Josefina Aparecida Pellegrini BRAGA ${ }^{1}$ \\ Élide Helena Guidolin da Rocha MEDEIROS ${ }^{1}$
}

\section{R E S U M O}

\section{Objetivo}

Identificar a proporção de anemia ferropriva e sua distribuição por estadiamento puberal, em pacientes atendidos em ambulatório de adolescência, e comparar os valores de corte habitualmente utilizados na adolescência para esse diagnóstico.

\section{Métodos}

Estudo retrospectivo, por análise de 453 prontuários dos pacientes atendidos de janeiro a dezembro de 2003, no Centro de Atendimento e Apoio ao Adolescente do Departamento de Pediatria da Universidade Federal de São Paulo, Escola Paulista de Medicina. Destes, 215 (47,0\%) rapazes e 238 (53,0\%) moças. Utilizaram-se os testes de Kappa e McNemar para avaliar as concordâncias e discordâncias entre dois critérios distintos; teste " $t$ " de Student para determinar as diferenças entre hemoglobina e índices hematimétricos nos grupos anêmico e não anêmico; e teste de Mann-Whitney para comparar os valores de hematócrito.

\section{Resultados}

A proporção de anemia, segundo os critérios adotados, foi de 7,50\% ( $n=34)$ e 6,84\% ( $n=31)$ na população; por estadiamento puberal encontrou-se, no sexo feminino, maior proporção nos estágios de mama 3 (30,00\%) e $4(50,00 \%)$ e no sexo masculino, em genitais $3(40,00 \%)$, havendo concordância entre os dois critérios.

\section{Conclusão}

Embora baixa, a proporção de anemia é relevante. A concordância obtida entre os valores propostos mostra que é possível usar qualquer um dos critérios. Há predomínio de anemia nas moças em estágio de mamas

\footnotetext{
1 Universidade Federal de São Paulo, Escola Paulista de Medicina, Departamento Pediatria. R. Botucatu, 715, 04023-062, São Paulo, SP, Brasil. Correspondência para/Correspondence to: M.S.S. VITALE. E-mail: <vitalle.dped@epm.br>.
} 
3 e 4, justificado pela maior necessidade de ferro e perdas menstruais, que ocorrem nessas fases, respectivamente; nos rapazes, a proporção maior em estágio 3 (fase de aceleração de crescimento) pode sugerir que eles já estavam depletados antes de entrar na puberdade.

Termos de indexação: anemia ferropriva; adolescente; puberdade; assistência ambulatorial.

\section{A B S T R A C T}

\section{Objective}

To identify the proportion and distribution of iron-deficiency anemia according to pubertal stage in patients seen at an outpatient clinic for adolescents and compare cutoff values normally used in adolescence for this diagnosis.

\section{Methods}

This retrospective study reviewed 453 medical records of patients seen from January to December 2003 at the Centro de Atendimento e Apoio ao Adolescente of the Pediatrics Department of the Federal University of São Paulo, Escola Paulista de Medicina (Adolescent Medicine Clinic from the Federal University of São Paulo). Of these, 215 (47.0\%) were males and 238 (53\%) were females. The Kappa and McNemar tests were used to assess the agreement and disagreement between two distinct criteria; the Student's " $t$ "-test was used to determine differences in hemoglobin and hematometric values between anemic and non-anemic patients; the Mann-Whitney test was used to compare hematocrit values.

\section{Results}

According to the adopted criteria, the proportion of anemia in the population was $7.50 \%(n=34)$ and $6.84 \%$ $(n=31)$. Considering pubertal stage and gender, a higher proportion was found among females with breast stage $3(30.00 \%)$ and $4(50.00 \%)$ and among males with genital stage $3(40.00 \%)$. Both criteria were in agreement.

\section{Conclusion}

The proportion of anemia is relevant, although low. The agreement obtained between the proposed values shows that it is possible to use any of the criteria. Anemia predominates among females with breast stages 3 and 4, justified by the higher need of iron and menstrual losses that occur in these stages, respectively; in males, the higher proportion in stage 3 (growth spurt) may suggest that iron stores were already depleted before they entered puberty.

Indexing terms: anemia, iron-deficiency; adolescent; puberty; ambulatory care.

\section{N T R O D U Ç Ã O}

A deficiência de ferro, e, especialmente, a anemia carencial ferropriva, representa uma das mais graves e importantes deficiências nutricionais que atinge, indiscriminadamente, países desenvolvidos ou em desenvolvimento ${ }^{1-3}$. Estima-se que cerca de dois bilhões de indivíduos, mais de 30,0\% da população mundial, sejam anêmicos, segundo dados da Organização Mundial de Saúde (OMS)4. Nos países em desenvolvimento calcula-se que $36,0 \%$ da população sofram de anemia nutricional, enquanto que a ocorrência nos países industrializados está em torno de 8,0\% ${ }^{4,5}$. Quando se considera a anemia por carência de ferro, têm-se os seguintes dados: 4,3\% a 20,0\% em países desenvolvidos, dependendo da idade e do sexo, contra $30,0 \%$ a $48,0 \%$ nos países em desenvolvimento ${ }^{1}$. As implicações negativas sobre os sistemas imune, endócrino, o crescimento físico e o desenvolvimento cognitivo que os indivíduos com anemia apresentam, fazem com que a anemia por deficiência de ferro seja grave problema de saúde pública'.

A adolescência é considerada, pela Organização Panamericana de Saúde, como um período sujeito a agravos ${ }^{6}$. Nessa fase da vida o indivíduo ganha em torno de $20,0 \%$ da sua estatura final e completa a sua maturação sexual ${ }^{7}$. Embora o adolescente adoeça pouco, a ingestão inadequada 
de alimentos, seja pela falta ou pelo excesso, é bastante comum, predispondo-o a alterações nutricionais ${ }^{8}$. Observa-se preferência pelo consumo de lanches e produtos ricos em açúcares e gorduras, em detrimento de alimentos ricos em vitaminas e minerais ${ }^{9}$.

O principal agravo nutricional na adolescência é a deficiência de micronutrientes, particularmente a de ferro, decorrente, principalmente, da ingestão inadequada, seja por modismos alimentares e/ou fatores socioeconômicos, em uma fase de grande necessidade de ferro para o crescimento, causada pelo aumento de incorporação de massa muscular, mais evidente nos rapazes, e por perdas menstruais irregulares, nas meninas ${ }^{9,10}$. Trabalho realizado na Suíça, com adolescentes maiores de 12 anos, evidenciou prevalência de anemia de $14,5 \%$ no sexo feminino e 7,9\% no sexo masculino ${ }^{11}$. Em alguns países europeus, como Espanha, Suécia e Inglaterra, a prevalência de anemia entre adolescentes foi, em média, 4,0\% ${ }^{12-14}$. Em nações em desenvolvimento essa situação é muito mais grave. Recente estudo realizado na Índia mostrou prevalência de anemia de $45,0 \%$ em adolescentes do sexo feminino ${ }^{15}$. $\mathrm{Na}$ Indonésia as prevalências foram 26,0\% e $11,0 \%$, quando estudados adolescentes dos sexos feminino e masculino, respectivamente ${ }^{16}$. Estudo realizado na Jamaica revelou que $25,0 \%$ dos adolescentes de 12 a 15 anos apresentavam-se anêmicos ${ }^{17}$.

No Brasil, algumas pesquisas regionais mostraram elevada prevalência de anemia ferropriva, especialmente entre adolescentes do sexo feminino. Em Minas Gerais esse distúrbio atingiu 16,6\% dos adolescentes entre 7 e 15 anos de idade ${ }^{18}$. Em São Paulo, Fujimori et al. ${ }^{19}$ encontraram $17,6 \%$ de prevalência de anemia em adolescentes do sexo feminino ${ }^{19}$. Em estudo realizado no Ambulatório de Adolescência Clínica do Núcleo de Nutrição, Alimentação e Desenvolvimento Infantil da Secretaria de Saúde do Estado de São Paulo foi observado que de 278 pacientes, $21,2 \%$ encontravam-se ferropênicos e 8,6\% anêmicos $^{20}$. Trabalho realizado em escola particular do Município de São Paulo detectou anemia em $11,0 \%$ dos adolescentes, o que foi considerado, pelos pesquisadores, prevalência baixa, mas acima da esperada por serem púberes de escola particular ${ }^{21}$.

Embora seja problema freqüente na adolescência, a literatura pouco descreve além da prevalência da anemia nessa faixa etária e suas conseqüências. Os trabalhos usam diferentes pontos de corte para diagnosticar anemia, o que dificulta a comparação ${ }^{22,23}$. Em nenhum deles se discute $\mathrm{O}$ motivo pelo qual tal valor de referência foi utilizado. Não foram encontrados trabalhos que questionem qual é o melhor valor de corte para esse diagnóstico, porém alguns, como Gallagher ${ }^{24}$ e OMS'1, são mais freqüentemente utilizados nessa faixa etária. Poucos trabalhos consistentes foram encontrados na literatura sobre a distribuição dos pacientes anêmicos em cada estadiamento de Tanner ${ }^{25}$. Diante do exposto, o trabalho tem como objetivos determinar a proporção de anemia carencial ferropriva e sua distribuição por estadiamento puberal na população de adolescentes atendidos no Centro de Atendimento e Apoio ao Adolescente (CAAA), disciplina de Especialidades Pediátricas da Universidade Federal de São Paulo (Unifesp), utilizando os valores de corte propostos por Gallagher e pela OMS, e discutir qual o melhor critério (Gallagher ou OMS) para detectar essa doença $a^{1,24}$.

\section{M É T O D O S}

Estudo retrospectivo, no qual foram revisados prontuários e compilados os dados de pacientes sem doença crônica, que haviam iniciado acompanhamento no ambulatório de adolescência do CAAA de janeiro a dezembro de 2003. Esse serviço atende adolescentes que vêm espontaneamente, encaminhados de outros serviços ou setores do Hospital São Paulo, Escola Paulista de Medicina. Os adolescentes são acompanhados dos 10 aos 20 anos de idade incompletos ${ }^{26}$, e, eventualmente, se atende pacientes de menor faixa etária, quando encaminhados; ou se prosse- 
gue o acompanhamento dos maiores de 20 anos, quando necessário.

Faz parte da rotina desse serviço solicitar hemograma para todos os pacientes que iniciam acompanhamento no ambulatório.

As informações coletadas nos prontuários foram: idade, sexo, estágio puberal, hemoglobina $(\mathrm{Hb})$, hematócrito $(\mathrm{Ht})$, hemoglobina corpuscular média (HCM) e volume corpuscular médio (VCM).

O estadiamento puberal foi realizado por médico treinado, que avaliou o desenvolvimento dos pêlos pubianos (P1, P2, P3, P4 e P5) em ambos os sexos, quanto às suas características, quantidade e distribuição; no sexo masculino considerou-se o estágio de evolução dos genitais ( $G 1, G 2, G 3, G 4$ e G5) e no feminino, o desenvolvimento das mamas (M1, M2, M3, M4 e M5), avaliados quanto ao tamanho, formas e características, segundo os critérios definidos por Tanner ${ }^{25}$.

Utilizaram-se, para definir anemia, os critérios propostos por Gallagher ${ }^{24}(\mathrm{Hb}<11,5 \mathrm{~g} / \mathrm{dL}$ e/ou $\mathrm{Ht}<35 \%$ para o sexo feminino e $\mathrm{Hb}<12 \mathrm{~g} / \mathrm{dL}$ e/ou $\mathrm{Ht}<35 \%$ para o sexo masculino), além das defi-nições da OMS', conforme Tabela 1. Para definir microcitose e hipocromia foram utilizados os se-guintes valores: $\mathrm{VCM}<79 \mathrm{fl}$ e $\mathrm{HCM}<26,5 \mathrm{pg}$.

Todos os exames foram realizados no Laboratório Central do Hospital São Paulo, por contagem eletrônica no Cell-Dyn 3000SL da Abbott. A dosagem de hemoglobina foi feita por absorbância pelo método de hemoglobina-cianeto. Além da hemoglobina esse aparelho determina o VCM. Os parâmetros complementares ( $\mathrm{Ht}, \mathrm{HCM})$ são obtidos por cálculos.

Tabela 1. Valores de hemoglobina e hematócrito abaixo dos quais anemia está presente, segundo a organização Mundial da Saúde.

\begin{tabular}{lccccc}
\hline \multirow{2}{*}{ Idade } & \multicolumn{2}{c}{ Hemoglobina $(\mathrm{g} / \mathrm{dL})$} & & \multicolumn{2}{c}{ Hematócrito $(\%)$} \\
\cline { 2 - 3 } \cline { 6 - 6 } & Meninas & Meninos & & Meninas & Meninos \\
\hline$<12$ anos & 11,0 & 11,0 & & 34 & 34 \\
$12 \vdash 15$ anos & 11,5 & 12,0 & & 34 & 35 \\
$15 \vdash 18$ anos & 11,7 & 12,3 & & 34 & 37 \\
18 anos $\vdash$ & 11,7 & 13,2 & & 34 & 39 \\
\hline
\end{tabular}

Considerou-se como critério de inclusão a presença dos valores de $\mathrm{Hb}$ e $\mathrm{Ht}$. Pacientes com doenças crônicas, que, sabidamente, alteram a série vermelha, foram excluídos do estudo.

Utilizaram-se os testes de Kappa e McNemar para avaliar a concordância ou discordância entre os critérios de Gallagher e da OMS.

Foram utilizados os testes " $t$ " de Student para determinar se havia significância entre a diferença dos valores de Hb, HCM e VCM dos pacientes não-anêmicos, quando comparados aos anêmicos, e Mann-Whitney para comparar os valores de Ht. Fixou-se em 0,05 o nível de rejeição da hipótese de nulidade, assinalando-se com asterisco os valores significantes.

O número de pacientes, no total geral de cada gráfico/tabela, é diferente devido às perdas ocorridas de cada variável. Considerando que se trata de levantamento de dados de prontuários, eventualmente, algumas informações encontram-se ausentes ou incompletas.

Este estudo está de acordo com a Resolução 196/96, do Conselho Nacional de Saúde do Ministério da Saúde, que regulamenta as pesquisas envolvendo seres humanos, tendo sido aprovado pelo Comitê de Ética em Pesquisa do Hospital São Paulo, Unifesp, nº 1572/05.

\section{RES U L T A D O S}

Foram estudados 453 prontuários referentes aos adolescentes que iniciaram, entre janeiro e dezembro de 2003, o acompanhamento no CAAA. Dos 453 estudados havia 215 (47,0\%) rapazes e 238 (53,0\%) moças.

A idade da população $(n=453)$ variou de 9 a 20 anos. A média de idade dos pacientes foi de 14,06 anos (sendo 14,20 nas meninas e 13,91 nos meninos). A distribuição da população por faixa etária foi a seguinte: 169 pacientes (37,3\%) tinham entre 13 a 14 anos; 139 (30,6\%) 11 a 12 anos; 97 (21,4\%) 15 a 16 anos; 33 (7,3\%) 17 a 
18 anos; $11(2,4 \%) 9$ a 10 anos e $4(1,0 \%) 19$ a 20 anos.

Dos 215 prontuários de rapazes havia informação sobre o estadiamento de Tanner, no que se refere aos genitais, em 173 (80,5\%). Desses, 27 (15,6\%) encontravam-se em G1; 46 $(26,6 \%)$ em G2; 41 (23,7\%) em G3; 46 (26,6\%) em G4 e $13(7,5 \%)$ em G5. No que se refere às moças, 195 (82,0\%) prontuários tinham informação sobre o estadiamento das mamas, sendo que, 6 (3,0\%) encontravam-se em M1; 22 (11,3\%) M2; $41(21,0 \%)$ M3; 97 (49,7\%) M4 e 29 (15,0\%) M5.

A proporção de anemia, segundo os critérios de Gallagher, foi 7,50\% ( $n=34)$, sendo $8,82 \%$ nas pacientes do sexo feminino $(n=21)$ e $6,04 \%$ nos do sexo masculino $(n=13)$. Ao serem usados os critérios propostos pela OMS, as proporções se alteram para $6,84 \%(n=31)$ na população geral, $7,56 \%(n=18)$ nas pacientes do sexo feminino e $6,04 \%(n=13)$ no sexo masculino.

As Tabelas 2, 3 e 4 mostram a concordância e discordância entre anêmicos e não-anêmicos, por Gallagher e OMS.

Quando os pacientes anêmicos $(n=34)$ foram classificados, de acordo com o estadiamento puberal de mamas e genitais, encontrou-se a seguinte distribuição: 2 (10\%) meninas encontravam-se em M1; 1 (5\%) em M2; 6 (30\%) em M3; 20 (50\%) em M4 e 1 (5\%) em M5; 2 (20\%) dos meninos estavam em $\mathrm{G} 1$; a mesma porcenta-

Tabela 2. Número de anêmicos e não-anêmicos utilizando os critérios propostos pela Organização Mundial de Saúde $(\mathrm{OMS})^{1}$ e por Gallagher ${ }^{24}$.

\begin{tabular}{lccc}
\hline \multirow{2}{*}{ Gallagher } & \multicolumn{3}{c}{ OMS } \\
\cline { 2 - 4 } & Anêmicos & Não-anêmicos & Total \\
\hline Anêmicos & 30 & 4 & 34 \\
Não-anêmicos & 1 & 418 & 419 \\
\hline Total & 31 & 422 & 453 \\
\hline
\end{tabular}

Teste Kappa: Kw=0,92; z calculado=19,54* $(p<0,001)$; Concordância diagonal=98,9\%/acima diagonal=0,88\%/abaixo diagonal=0,22\%. Teste McNemar: $\chi^{2}$ calculado=0,80 (NS). gem em G2 e G4; 5 (40\%) em G3 e nenhum em G5.

A Tabela 5 mostra as médias e os desvios-padrão (DP) dos índices hematimétricos da população estudada. O grupo não-anêmico apresentou média de HCM e VCM significantemente maior do que o grupo anêmico.

Comparando as médias dos valores de $\mathrm{Ht}$ entre os grupos anêmico e não-anêmico, observou-se que o grupo não-anêmico apresentou média $(40,68 \%)$ significantemente maior do que o grupo anêmico (33,54\%), $p<0,001$.

Tabela 3. Número de anêmicos e não-anêmicos, segundo os critérios da Organização Mundial de Saúde ${ }^{1}$ e de Gallagher ${ }^{24}$, nas pacientes do sexo feminino, em cada estadiamento puberal de Tanner. São Paulo, 2003.

\begin{tabular}{|c|c|c|c|c|}
\hline \multirow{2}{*}{ Testes } & \multicolumn{4}{|c|}{ OMS } \\
\hline & Gallagher & Anêmicos & Não-anêmicos & Total \\
\hline \multirow[t]{3}{*}{ M1 } & Anêmicos & 2 & 0 & 2 \\
\hline & Não-anêmicos & 0 & 4 & 4 \\
\hline & Total & 2 & 4 & 6 \\
\hline \multirow[t]{3}{*}{ M2 } & Anêmicos & 1 & 0 & 1 \\
\hline & Não-anêmicos & 0 & 21 & 21 \\
\hline & Total & 1 & 21 & 22 \\
\hline \multirow[t]{3}{*}{ M3 } & Anêmicos & 4 & 2 & 6 \\
\hline & Não-anêmicos & 0 & 35 & 35 \\
\hline & Total & 4 & 37 & 41 \\
\hline \multirow[t]{3}{*}{ M4 } & Anêmicos & 8 & 2 & 10 \\
\hline & Não-anêmicos & 0 & 87 & 87 \\
\hline & Total & 8 & 89 & 97 \\
\hline \multirow[t]{3}{*}{ M5 } & Anêmicos & 1 & 0 & 1 \\
\hline & Não-anêmicos & 0 & 28 & 28 \\
\hline & Total & 1 & 28 & 29 \\
\hline
\end{tabular}

M1: Teste Kappa: $K w=1,00$; $z$ calculado $=2,44^{*}(p<0,001)$; Concordância diagonal $=100 \%$. Teste McNemar: $\chi^{2}$ calculado $=0,0$.

M2: Teste Kappa: Kw=1,00; z calculado $=4,69^{*}(p<0,001)$; Concordância diagonal $=100 \%$. Teste McNemar: $\chi^{2}$ calculado $=0,0$.

M3: Teste Kappa: $\mathrm{Kw}=0,77$; z calculado $=5,09 *(p<0,001)$; Concordância diagonal=95,1\% /acima diagonal=4,9\% abaixo diagonal $=0,0$. Teste McNemar: $\chi^{2}$ calculado $=0,50$ (NS).

M4: Teste Kappa: Kw=0,88; z calculado $=8,71 *(p<0,001)$; Concordância diagonal=97,9\% /acima diagonal=2,1\% abaixo diagonal $=0,0$. Teste McNemar: $\chi^{2}$ calculado=0,50 (NS).

M5: Teste Kappa: $K w=1,0 ; z$ calculado=5,38* $(p<0,001)$; Concordância diagonal $=100 \%$. Teste McNemar: $\chi^{2}$ calculado $=0,0$. 


\section{DIS C U S S Ã O}

Há poucos dados disponíveis sobre a prevalência de anemia ferropriva na adolescência, embora ela pareça ser elevada, principalmente, nas moças $2,11,15,16,21$. Os trabalhos existentes res-

Tabela 4. Número de anêmicos e não-anêmicos pelos critérios propostos pela OMS ${ }^{1}$ e Gallagher ${ }^{24}$, nos pacientes do sexo maculino, em cada estadiamento puberal de Tanner.

\begin{tabular}{|c|c|c|c|c|}
\hline \multirow{2}{*}{ Testes } & \multicolumn{4}{|c|}{ OMS } \\
\hline & Gallagher & Anêmicos & Não-anêmicos & Total \\
\hline \multirow[t]{3}{*}{ G1 } & Anêmicos & 2 & 0 & 2 \\
\hline & Não anêmicos & 0 & 25 & 25 \\
\hline & Total & 2 & 25 & 27 \\
\hline \multirow[t]{3}{*}{ G2 } & Anêmicos & 2 & 0 & 2 \\
\hline & Não anêmicos & 0 & 44 & 44 \\
\hline & Total & 2 & 44 & 46 \\
\hline \multirow[t]{3}{*}{ G3 } & Anêmicos & 5 & 0 & 5 \\
\hline & Não anêmicos & 0 & 36 & 36 \\
\hline & Total & 5 & 36 & 41 \\
\hline \multirow[t]{3}{*}{ G4 } & Anêmicos & 2 & 0 & 2 \\
\hline & Não anêmicos & 0 & 44 & 44 \\
\hline & Total & 2 & 44 & 46 \\
\hline \multirow[t]{3}{*}{ G5 } & Anêmicos & 0 & 0 & 0 \\
\hline & Não anêmicos & 0 & 13 & 13 \\
\hline & Total & 0 & 13 & 13 \\
\hline
\end{tabular}

G1: Teste Kappa: $K w=1,00 ; z$ calculado=5,19* $(p<0,001)$; Concordância diagonal=100\%; Teste McNemar: $\chi^{2}$ calculado $=0,0$.

G2: Teste Kappa: $K w=1,00 ; z$ calculado=6,78* $(p<0,001)$; Concordância diagonal $=100 \%$; Teste McNemar: $\chi^{2}$ calculado $=0,0$.

G3: Teste Kappa: KW=1,0; z calculado=6,40* $(p<0,001)$; Concordância diagonal=100\%; Teste McNemar: $\chi^{2}$ calculado $=0,0$.

G4: Teste Kappa: Kw=1,0; z calculado=6,78* $(p<0,001)$; Concordância diagonal=100\%; Teste McNemar: $\chi^{2}$ calculado $=0,0$.

G5: Teste Kappa: Kw=1,0; z calculado $=5,35^{*}(p<0,001)$; Concordância diagonal=100\%; Teste McNemar: $\chi^{2}$ calculado=0,0. tringem-se a pequenas populações e a ambulatórios. Faltam trabalhos multicêntricos e levantamentos nacionais, o que dificulta a comparação e o conhecimento da real prevalência de anemia nessa faixa etária.

Devido às mudanças nas necessidades nutricionais dos adolescentes, ao início da menstruação nas garotas, às modificações hormonais decorrentes da puberdade, há diferenças na concentração de hemoglobina entre os sexos, em idades diferentes e em estadiamentos diferentes, considerando-se adolescentes pré-púberes e aqueles já púberes. Porém, há poucos trabalhos disponíveis a respeito da relação entre puberdade e níveis de hemoglobina, tanto em um sexo quanto no outro ${ }^{21,27}$.

O número de trabalhos na literatura, que relaciona anemia na adolescência com estadiamento puberal, vem aumentando. Apesar disso, observa-se nítida dificuldade em estabelecer qual o melhor parâmetro puberal a ser utilizado, se mamas e genitais (considerando moças e rapazes) ou pêlos. Em alguns trabalhos, o estado puberal foi aferido baseando-se no método do status quo, no qual se perguntou à adolescente ou ao adolescente se havia, respectivamente, apresentado menarca ou polução noturna ${ }^{28,29}$. Um dos trabalhos utilizou a média entre mamas e pêlos pubianos nas meninas, e genitais e pêlos pubianos nos meninos ${ }^{30}$; em outro, por exemplo, foi utilizado "o menor estágio assinalado pelo adolescente", quando a esse era solicitado classificar as suas mamas e pêlos (meninas) ou seus genitais e pêlos $(\text { meninos })^{21}$.

A proporção de anemia encontrada, embora pequena (7,54\% por Gallagher e 6,84\%

Tabela 5. Análise estatística dos índices hematimétricos da população de anêmicos e não-anêmicos pelos critérios de Gallagher.

\begin{tabular}{|c|c|c|c|c|c|c|c|c|c|c|}
\hline \multirow{2}{*}{$\begin{array}{l}\text { Índices } \\
\text { hematimétricos }\end{array}$} & \multicolumn{4}{|c|}{ Anêmicos } & \multicolumn{4}{|c|}{ Não-anêmicos } & \multicolumn{2}{|c|}{ Análise estatística } \\
\hline & $\mathrm{n}$ & M & DP & Amplitude & $n$ & M & DP & Amplitude & $t$ & $p$ \\
\hline $\mathrm{Hb}(\mathrm{g} / \mathrm{dL})$ & 34 & 10,77 & 1,10 & $6,80-12,00$ & 419 & 13,55 & 1,08 & $11,50-17,60$ & 14,17 & $<0,001$ \\
\hline HCM (pg) & 30 & 24,78 & 3,85 & $14,53-31,00$ & 359 & 28,11 & 2,01 & $21,00-35,80$ & 4,67 & $<0,001$ \\
\hline VCM (fl) & 32 & 75,92 & 9,66 & $50,94-92,60$ & 364 & 84,48 & 5,64 & $66,60-128,00$ & 4,93 & $<0,001$ \\
\hline
\end{tabular}

$\mathrm{n}=$ número; $\mathrm{M}=$ média; $\mathrm{DP}=$ desvio-padrão; $t=$ teste " $\mathrm{t}$ " de Student; $p=$ nível de significância. 
pela OMS), quando comparada a estudos realizados em outros países em desenvolvimento, é relevante, pois pode ser causa de inúmeras repercussões. A deficiência de ferro, com ou sem anemia, interfere no aprendizado, no apetite e na capacidade de trabalho; podendo também ser fator limitante para o crescimento e bom desempenho escolar na adolescência ${ }^{31}$. É provável que o baixo percentual de anemia encontrado seja decorrente do tipo de paciente que é atendido no serviço. Geralmente, os pacientes são referendados pelos Centros de Saúde da região ou pelos ambulatórios de pediatria geral e especialidades pediátricas do Hospital São Paulo, sendo, portanto, pacientes que vêm sendo assistidos. Isso sugere que, talvez, os índices de anemia em população desta idade, sem acompanhamento, sejam maiores do que o encontrado.

A literatura não apresenta consenso em relação a qual critério utilizar para definir anemia nessa faixa etária; e, ao estudar esse evento, se depara com trabalhos de difícil comparação, pela utilização de pontos de corte diferentes, sendo os mais utilizados os propostos por Gallagher e pela OMS. As Tabelas 2 a 4 mostram que não há diferença quando se utiliza qualquer um desses critérios, mesmo quando se relaciona com o estadiamento puberal de Tanner. A sensibilidade de ambos é a mesma.

Na avaliação do desenvolvimento puberal deu-se importância, apenas, para mamas, nas moças, e para os genitais, nos rapazes, pois, embora exista um padrão de desenvolvimento pubertário em relação à pilificação, a variabilidade desse parâmetro é muito maior do que a avaliação por mamas e genitais; sendo que a pilificação reflete a atividade do eixo hipotálamo-hipófise-gonadal/ adrenal, e as mamas e os genitais refletem a atividade do eixo hipotálamo-hipófise-gonadal, não devendo, portanto, as variáveis pêlo e mamas/ genitais serem "misturadas" na avaliação do estadiamento puberal.

Embora não fosse objetivo desse trabalho, chamou atenção a diferença na distribuição pubertária dos pêlos, encontrada nos pacientes anê- micos e nos não-anêmicos (dados não apresentados), mostrando que é melhor trabalhar com genitais e mamas, ao invés de pêlos. No grupo não-anêmico, a pilificação seguiu um padrão de distribuição pubertário, e no anêmico houve "perda" desse padrão. Esse resultado talvez se deva à influência da mistura de raças em nosso meio; ou se poderia supor que, na vigência de anemia, o eixo hipotálamo-hipófise-gonadal/ adrenal sofra alguma alteração.

Apesar de não se conhecer muito a respeito da prevalência de anemia por deficiência de ferro entre adolescentes do sexo masculino, alguns trabalhos mostram que a baixa prevalência entre os rapazes, em fase pubertária, pode ser explicada pelo aumento fisiológico da concentração de hemoglobina, causado pela maturação sexual, assim como pela diminuição das necessidades depois de completado o estirão pubertário ${ }^{27,29}$. 0 pico do crescimento pubertário nas moças ocorre, aproximadamente, um ano antes da menarca podendo, o crescimento rápido, ser causa de anemia. A puberdade na menina aumenta o risco de anemia, enquanto diminui nos rapazes (considerando-se puberdade a menarca nas moças e a polução noturna nos rapazes $)^{29}$. No sexo feminino observou-se mais anemia nos estágios M3 (30\%) - pico do estirão, e M4 (50\%) - fase de desaceleração do crescimento. A justificativa, possivelmente, se encontre no aumento das necessidades, que acontece no pico do estirão, e nas perdas menstruais, muitas vezes, irregulares e abundantes, na desaceleração do crescimento. Nos rapazes encontrou-se $40 \%$ de anemia no estágio G3, portanto, na fase de aceleração do crescimento. Considerando que no pico da aceleração (G4) ocorre maior aporte de massa magra, esse resultado sugere que esses rapazes já vinham apresentando deficiência de ferro anteriormente. Em trabalho realizado com adolescentes de escola privada, na cidade de São Paulo, houve maior prevalência de anemia em adolescentes púberes, além disso se observou associação entre estágios de maturação sexual e presença de anemia somente nas meninas (prevalências maiores em estágios 3$)^{21}$. 
Para o diagnóstico preciso de anemia por deficiência de ferro, testes de triagem compreendendo a contagem sangüínea, VCM e HCM deveriam acompanhar os testes confirmatórios de deficiência de ferro no sangue e/ou nas reservas de ferro. A OMS recomenda que a etiologia da deficiência de ferro seja avaliada pela presença de dois ou mais parâmetros laboratoriais alterados $^{1}$.

Muitos estudos brasileiros usam somente os níveis de hemoglobina na definição metodológica para investigar anemia, talvez, porque não há a preocupação em caracterizar a sua etiologia, provavelmente, pelo fato de a anemia por deficiência de ferro ser a maior causa de anemia em todo o mundo, sobretudo, nos países em desenvolvimento, além das dificuldades operacionais em definir as demais quantificações, desde falta de verba, até inadequado preenchimento de prontuários médicos.

A reconhecida diferença na concentração de hemoglobina em diferentes raças - a negra tem valor de hemoglobina, sabidamente, menor do que a branca - torna as curvas existentes inadequadas para o Brasil, onde a população é ricamente miscigenada. Portanto, novos estudos são necessários para estabelecer padrões de referência de valores de hemoglobina em populações com muita miscigenação ${ }^{32-34}$.

A Tabela 5 mostra que, efetivamente, classificação da anemia encontrada neste trabalho é a do tipo hipocrômica e microcíticas, corroborando a idéia de anemia carencial. O teste " $t$ " de Student evidenciou que as médias de $\mathrm{Hb}, \mathrm{HCM}$ e VCM dos pacientes anêmicos e não-anêmicos são significantemente diferentes.

Os valores de $\mathrm{Ht}$ foram significantemente menores no grupo anêmico, sendo que a média encontrada (33,54\%) está abaixo do ponto de corte estabelecido por Gallagher (35,00\%) e pela OMS $(34,00 \%$ a $39,00 \%)$. Alguns pacientes apresentavam $\mathrm{Ht}$ abaixo do valor de corte e $\mathrm{Hb}$ dentro da faixa de normalidade. Esses indivíduos também são considerados anêmicos. Enfatiza-se, então, a necessidade da avaliação, também, do hematócrito para o estabelecimento de anemia.
Vale observar que no grupo não-anêmico, $37(8,7 \%)$ pacientes apresentavam $\mathrm{Ht} \geq 45 \%$ $(45,0 \%$ a $54,9 \%)$. Eram 8 (22,0\%) do sexo feminino, em estadiamentos M2, M3 e M4, e 29 $(78,0 \%)$ do sexo masculino, em estadiamentos G3, G4 e G5. Nenhum desses pacientes era doente pulmonar crônico, cardiopata ou tabagista, mostrando, portanto, a ação androgênica, que se torna evidente nos estadiamentos mais avançados, principalmente, no sexo masculino.

\section{O N CLUSÃ O}

Embora baixa, a proporção de anemia é relevante. A concordância obtida entre os valores propostos por Gallagher e pela OMS mostra que é possível usar qualquer um deles, até quando se relaciona com o estadimento puberal, tornando, portanto, os trabalhos que utilizam esses critérios comparáveis entre si. Há maior predomínio de anemia nas moças em estágio de mamas 3 e 4 , justificado pela maior necessidade de ferro presente no estirão, e pelas perdas menstruais, que ocorrem nessas fases, respectivamente; nos rapazes, a proporção maior em estágio 3 (fase de aceleração de crescimento) pode sugerir que eles já estavam depletados antes de entrar na puberdade.

Sabidamente, a anemia é altamente prevalente na faixa etária pediátrica e, muitas vezes, não há aderência ao tratamento, ou o meio ambiente não propicia a cura (condições inadequadas de saneamento básico, má alimentação). Portanto, uma questão a ser pensada é até que ponto o indivíduo já depletado de ferro (anêmico ou não) na infância influenciará o evoluir dos eventos pubertários.

Recomenda-se, ao médico cuidador de adolescentes, atenção não só ao momento de eclosão dos eventos pubertários e à sua evolução, como também, à possibilidade de ocorrer deficiência de ferro nessa faixa etária, antes mesmo do aparecimento dos sinais e sintomas clínicos de anemia. 


\section{COLABORADORES}

F.C. SILVA e E.C. QUAGLIA participaram da coleta de dados, levantamento bibliográfico, análise estatística e redação científica. M.S.S. VITALLE delineou o estudo, coleta de dados, levantamento bibliográfico, análise estatística e redação científica. J.A.P. BRAGA e E.H.G.R. MEDEIROS participaram da análise estatística e redação científica.

\section{REFER Ê NCIAS}

1. World Health Organization. Iron deficiency anaemia: assessment prevention and control. A guide for programme managers. Geneva, 2001 [cited 2005 Aug 15] Avaliable from: URL: http:// www.who.int/nut/publications.htm

2. Frith-Terhune AL, Cogswell ME, Kettel KL, Will JC, Ramakrishna U. Iron deficiency anemia: higher prevalence in Mexican American than in nonHispanic females in the third National Health and Nutrition Examination Survey, 1988-1994. Am J Clin Nutr. 2000; 72(4):963-8.

3. Monteiro CA, Szarfarc SC, Mondini L. Tendência secular da anemia na infância na cidade de São Paulo (1984-1996). Rev Saúde Pública. 2000; 34(6):62-72.

4. World Health Organization. Micronutrient deficiencies: batting iron deficiency anaemia. Geneva, 2002 [cited 2005 Aug 15]. Avaliable from: URL: http://www.who.int/nut/ida.htm

5. Mora JO, Mora OL. Deficiencias de micronutrientes en América Latina y el Caribe: anemia ferropriva. Washington (DC): OPS; 1998.

6. Lopez G, Yunes J, Solés JA, Omran AR. Salud Reprodutiva en las Americas. Washington (DC): OPS; 1992.

7. Barnes HV. Physical growth and development during puberty. Med Clin North Am. 1975; 59(6): 1305-17.

8. Tojo R, Leis R, Pavon P. Necesidades nutricionales en la adolescência. Factores de riesgo. An Esp Pediatr. 1992; 49(36 Suppl):80-5.

9. Gambardella AMD, Frutuoso MFP, Franchi C. Prática alimentar de adolescentes. Rev Nutr. 1999; 12(1): 55-63.

10. Slap GB, Khalid N, Paikoff RL, Brooks-Gunn J, Warren MP. Evolving self-image, puberal manifestations and puberal hormones: preliminary findings in young adolescent girl. J Adolesc Health. 1994; 15(4):327-35.
11. Massawe SN, Ronquist G, Nystrõm L, Lindmark G. Iron status and iron deficiency anaemia in adolescents in a Tanzanian suburban area. Gynecol Obstet Invest. 2002; 54(3):137-44.

12. Caballo-Roig N, Garcia P, Valdemoro M, Del Castillo ML, Tapia MS, Gonzalez Vargaz A, et al. The prevalence of anemia in the children and adolescents of Madrid. An Esp Pediatr. 1993; 39(3): 219-22.

13. Hallberg L, Hulten L, Lindstedt G, Lundberg PA, Mark A, Purins J, et al. Prevalence of iron deficiency in Swedish adolescents. Pediatric Res. 1993; 34(5): 680-7.

14. Nelson M, White J, Rhodes C. Haemoglobin ferritin, and iron intakes in British children aged 12-14 years: a preliminary investigation. Br J Nutr. 1993; 70(1):147-55.

15. Das DK, Biswas R. Nutritional status of adolescents girls in a rural area of North 24 Paraganas district, West Bengal. Indian J Public Health. 2005; 49(1): 18-21.

16. Soekarjo DD, Pee Sd S, Kusin JA, Schreurs WH, Schultink W, et al. Effectiveness of weekly vitamin A $(10,000 \mathrm{IU})$ and iron $(60 \mathrm{mg})$ suplementation for adolescent boys and girls through schools in rural and urban East Java, Indonésia. Eur J Clin Nutr. 2004; 58(6):927-37.

17. Graciano F. Integrated iron supplementation for women: a new approach for iron deficiency control. Yakarta: SEAMEO/TROPMED; 1999.

18. Norton RC, Figueiredo RC, Diamante R, Goulart $E M$, Mota JA, Viana MB, et al. Prevalence of anemia among school-children from Rio Acima (State of Minas Gerais, Brazil): use of the standardized prevalence method and evaluation of iron deficiency. Braz J Med Biol Res. 1996; 29(12): 1617-24.

19. Fujimori E, Szarfarc S, Oliveira IM. Prevalence of iron deficiency anemia in female adolescents in Taboão da Serra, SP. Brazil. Rev Latinoam Enfermagem. 1996; 4(3):49-63.

20. Vitalle MSS, Queiroz SS. Anemia carencial ferropriva. In: Costa COM, Souza RP. Adolescência aspectos clínicos e psicossociais. São Paulo: Artmed; 2002. p.30-7.

21. Juliano BA, Frutuoso MFP, Gambardella AMD. Anemia em adolescentes segundo maturação sexual. Rev Nutr. 2004; 17(1):37-43.

22. Current Trends CDC Criteria for anaemia in children and childbearing-aged women. Morb Mortal Weekly Rep (MMWR). 1989; 38(22):400-4.

23. Demayer E, Dallman PR, Gerney JM, Hallberg L, Sood SK, Srikantia SG. Preventing and controlling iron deficiency anemia through primary health care. Geneve: WHO; 1989. 
24. Gallagher JR, Heald FP, Garrel DC. Medical care of the adolescent. 3rd ed. New York: Appleton; 1976.

25. Tanner JM, Whitehouse RH. Clinical longitudinal standarts for height, weight, height velocity, weight velocity and stages of puberty. Arch Dis Child. 1976; 51(3):170-9.

26. Organización Mundial de la Salud. El embaraço y el aborts en la adolescência. Geneve: OMS; 1975. Série de Informes Técnicos, 583.

27. Anttila R, Siimes MA. Serum transferrin and ferritin in pubertal boys: relations to body growth, pubertal age, erythropoesis, and iron deficiency. Am J Clin Nutr. 1996; 63(2):179-83.

28. Cameron N. Growth and maturation data during adolescence. The use and interpretation of anthropometry in adolescents. Geneva: WHO; 1993.

29. Soekarjo DD, Pee S, Bloem MW, Tjiong R, Yip R, Schreurs WHP, et al. Socio-economic status and puberty are the main factors determining anaemia in adolescent girls and boys in East Java, Indonesia. Eur J Clin Nutr. 2001; 55(11):932-9.

30. Frutuoso MFP, Vigantzky VA, Gambardella AMD. Níveis séricos de hemoglobina em adolescentes segundo estágio de maturação sexual. Rev Nutr. 2003; 16(2):155-62.

31. Bruner AB, Joffe A, Duggan AK, Casella JF, Brandt J. Randomised study of cognitive effects of iron supplementation in non-anaemic iron-deficient adolescent girls. Lancet. 1996; 348(9033):992-6.

32. Dallman PR, Barr GD, Allen CM, Shinefield MD. Hemoglobin concentration in white, black and oriental children: is there a need for separate criteria in screening for anemia? Am J Clin Nutr. 1978; 31(3):377-80.

33. Reeves JD, Driggers DA, Lo EY, Dallman PR. Screening for anemia in infants: evidence in favor of using identical hemoglobin criteria for blacks and Caucasians. Am J Clin Nutr. 1981; 34(10): 2154-7.

34. Perry GB, Byers T, Yip R, Margen S. Iron nutrition does not account for the hemoglobin differences between blacks and whites. J Nutr. 1992; 122(7): 1417-24.

Recebido em: 29/9/2005

Versão final reapresentada em: 30/10/2006 Aprovado em: 22/12/2006 